\title{
Parallel Arithmetical Unscented Kalman Filter Technic for Lithium-ion Battery State-of-Charge Estimation

\author{
WeilongLiu ${ }^{1,2, a}$,LiyeWang ${ }^{1, b}$, LifangWang $^{1, c}$ and ChenglinLiao ${ }^{1, d}$
}

${ }^{1}$ Key Laboratory of Power Electronics and Electric Drives, Institute of Electrical Engineering, Chinese Academy of Science, Beijing, China

${ }^{2}$ University of Chinese Academy of Sciences, Beijing, China

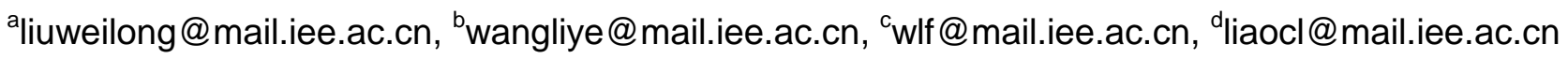

Keywords:Lithium-ion battery; State-of-Charge(SOC); Battery model; Parallel arithmetic;Unscented Kalman filter(UKF)

Abstract.In order to apply the lithium-ion batteries on the electric vehicles reliably and safely, estimating the internal statements of the batteries, such as the state-of-charge (SOC) is obligatory. The purpose of this work was to present a SOC estimation method named parallel arithmetical unscented Kalman filter which has obvious advantages that was validated by simulation. In this paper, a lumped circuit model and the parameter identification method were studied. And the state-space type battery model was derived. Then a novel SOC estimation method was proposed using the parallel arithmetical unscented Kalman filter (PAUKF) technique. Validation results showed that the presented SOC estimation algorithm could have an acceptable performance with the mean error less than $2.4 \%$.

\section{Introduction}

Nowadays, new energy automobiles, such as blade electric vehicle, hybrid electric vehicle, fuel cell electric vehicle, are promoted successfully among which the blade electric vehicles and hybrid electric vehicles share the most part of the market. And the lithium-ion batteries are the most commonly used as the power batteries. The lithium-ion batteries need to be operated in the mild conditions, which can be estimated by the specific algorithms, in which the SOC is the essential statement that most engineers and car drivers care about. But the SOC can't be measured and only can be estimated.

A plenty of SOC estimation algorithms were proposed which could be divided into three aspects: First, the traditional estimation algorithms include the coulomb counting method[1], which is short of the accumulative error, and open circuit voltage rectification method[2], which can't be operated in the real-time. Second, the empirical-based estimation algorithmsapplied techniques of the neural network[3], support vector machine[4], fuzzy logic[5], etc., which must be trained with numerous empirical data and can hardly achieve high accuracy. Third, the model-based estimation algorithms, which utilized equivalent lumped circuit[6,7] or battery mechanism equations[8,9] as the battery model, have been widely used on the electric vehicles because of the convenience application in the real-time.

In this work, a novel SOC estimation algorithm was proposed, which was based on the technic of parallel arithmeticalunscented Kalman filter (PAUKF). The simulation comparison was made between the PAUKF and the adaptive extended Kalman filter (AEKF) to validate the accuracy and preponderance over PAUKF.

\section{Battery modeling and parameter identification}

\section{Modeling of Battery}

There are kinds of battery models have been proposed, such as the equivalent lumped circuit models (ELCM), which includethe Rint model, Thevenin model and DP model, etc.[10], electrochemicalmechanism models. Because the ELCM can make a good balance between accuracy and computational complexity, the Thevenin model was applied for the estimation of SOCin this 
paper. Fig. 1 shows the schematic of the Thevenin model, in which $U_{o c}$ is the ideal open circuit voltage, $U_{T}$ is the terminal voltage, $R_{o}$ is the internal resistance, $R_{p}$ is the polarization resistance and $C_{p}$ is the polarization capacitance, $I_{L}$ is the current which is positive when the battery is discharged.

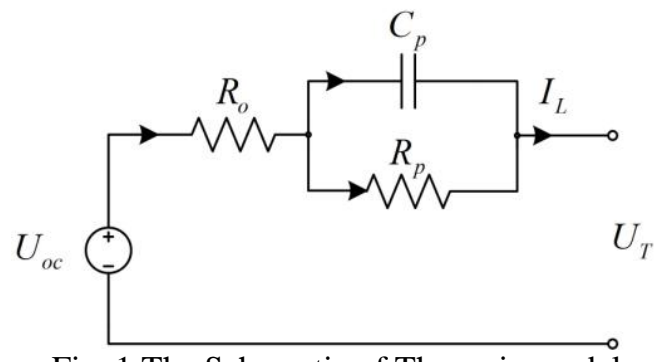

Fig. 1 The Schematic of Thevenin model

The Thevenin model equations can be derived as (1).

$$
\left\{\begin{array}{l}
\dot{U}_{p}=-\frac{1}{R_{p} C_{p}} U_{p}+\frac{1}{C_{p}} I_{L} \\
U_{T}=U_{o c}-U_{p}-R_{o} I_{L}
\end{array}\right.
$$

Because the battery experiment data is discrete, the battery model must be derived into discrete form in order to apply the input and output experiment data of the battery. The discrete form of the battery model at the sample time $k$ was derived as(2). $\Delta t$ is the sampleinterval.

$$
\left\{\begin{array}{l}
U_{p, k}=e^{-\frac{\Delta t}{R_{p} C_{p}}} \times U_{p, k-1}+\left(1-e^{-\frac{\Delta t}{R_{p} C_{p}}}\right) \times I_{L, k} R_{p} .(2) \\
U_{T, k}=-U_{p, k}-R_{o} I_{L, k}+U_{o c, k}
\end{array}\right.
$$

\section{Parameter Identification}

Before the SOC estimation, the relationship between the battery model parameters and SOC must be identified. In this work, the experiment object was a $12 \mathrm{Ah}$ lithium-ion ferrous phosphate battery. The experiment procedure was: 1 . discharging the battery to $0 \%$ SOC; 2 . resting the battery for 2 hours every time when the battery was charged $5 \%$ SOC with $0.5 \mathrm{C}$ charging current until the battery was fully charged; 3 . resting the battery for 2 hours every time when the battery was discharged $5 \%$ SOC with $0.5 \mathrm{C}$ discharging current until the battery was fully discharged; 4 . getting the mean value of $U_{o c}$ and $R_{o}$ through calculation; 5. achieving the $R_{p}$ and $C_{p}$ using the least square fitting method. The fitting equations of battery model parameters with SOC were shown as (3).

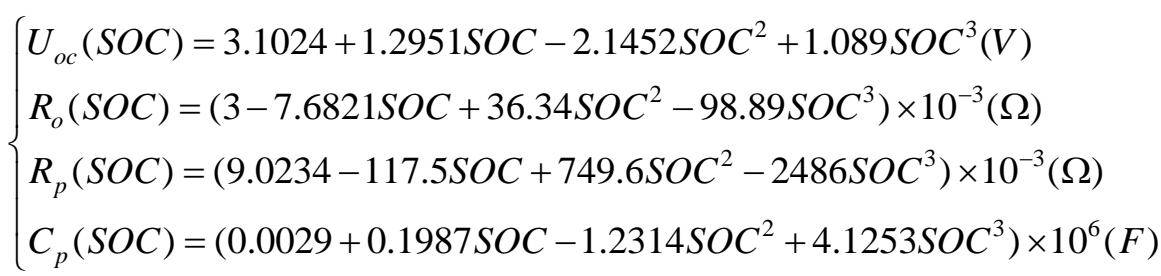

\section{SOC estimation algorithm}

\section{State-space of Battery Model}

The SOC of the battery could be achieved by coulomb counting, as shown in (4). $\eta$ is the coulombic efficiency. $\Delta t$ is the sample interval. $k$ is the sample time. $C_{B}$ is the capacity of the battery. 


$$
\operatorname{SOC}(k)=\operatorname{SOC}(k-1)-\frac{\eta \Delta t I_{L}(k-1)}{3600 C_{B}}
$$

Combining the (2) and (4), the state equations and observation equation could be derived into matrix form as shown in (5) and (6). $\omega \sim N(0, Q)$ is the process noise. $v \sim N(0, R)$ is the measurement noise. $\operatorname{Cov}(\omega, v)=0$.

$$
\begin{aligned}
& \left(\begin{array}{l}
U_{p, k} \\
S O C_{k}
\end{array}\right)=\left(\begin{array}{cc}
e^{-\frac{\Delta t}{R_{p} C_{p}}} & 0 \\
0 & 1
\end{array}\right)\left(\begin{array}{l}
U_{p, k-1} \\
S O C_{k-1}
\end{array}\right)+\left(\begin{array}{c}
R_{p}\left(1-e^{-\frac{\Delta t}{R_{p} C_{p}}}\right) \\
\frac{\eta \Delta t}{3600 C_{B}}
\end{array}\right) I_{L, k-1}+\left(\begin{array}{l}
\omega_{p, k-1} \\
\omega_{S O C, k-1}
\end{array}\right) \cdot(5) \\
& U_{T, k}=\left(-1 \frac{U_{o c, k}-R_{o} I_{L, k}}{S O C_{k}}\right)\left(\begin{array}{l}
U_{p, k} \\
S O C_{k}
\end{array}\right)+v_{k} \cdot(6)
\end{aligned}
$$

\section{AEKF and Its Shortages}

For using the AEKF, the state equations of the battery model were linearized and the matrix form was shown as (7).

$$
\left(\begin{array}{l}
U_{p, k} \\
S O C_{k}
\end{array}\right)=\left(\begin{array}{cc}
1-\frac{\Delta t}{R_{p} C_{p}} & 0 \\
0 & 1
\end{array}\right)\left(\begin{array}{l}
U_{p, k-1} \\
S O C_{k-1}
\end{array}\right)+\left(\begin{array}{c}
\frac{\Delta t}{C_{p}} \\
\frac{\eta \Delta t}{3600 C_{B}}
\end{array}\right) I_{L, k-1}+\left(\begin{array}{l}
\omega_{p, k-1} \\
\omega_{S O C, k-1}
\end{array}\right) \cdot(7)
$$

The linearized Thevenin model was shown as (8).

$$
\left\{\begin{array}{l}
x_{k}=f\left(x_{k-1}, u_{k-1}\right)+\omega_{k-1} \approx A_{k-1} x_{k-1}+B_{k-1} u_{k-1}+\omega_{k-1} \\
y_{k}=h\left(x_{k}, u_{k}\right)+v_{k} \approx C_{k} x_{k}+v_{k}
\end{array} .\right.
$$

With $x_{k}=\left(\begin{array}{l}U_{p, k} \\ S O C_{k}\end{array}\right), y_{k}=U_{T k}, A_{k}=\left(\begin{array}{cc}1-\frac{\Delta t}{R_{p} C_{p}} & 0 \\ 0 & 1\end{array}\right), B_{k}=\left(\begin{array}{c}\frac{\Delta t}{C_{p}} \\ \frac{\eta \Delta t}{3600 C_{B}}\end{array}\right), C_{k}=\left(\begin{array}{c}-1 \frac{U_{o c, k}-R_{o} I_{L, k}}{S O C_{k}}\end{array}\right)$.

Observed that $C_{k}$ was meaningless if $S O C_{k}$ was zero, so the variable substitution method was applied that $U_{o c}\left(S O C_{k}\right)$ was substituted with $U_{o c}\left(S O C_{k}-S O C_{0}\right)$, which $S O C_{0}$ is positive.

The procedure of AEKF was shown in (9) (10).

Initialize with:

$$
x_{0}, P_{0}, Q_{0}, R_{0}(9)
$$

Prediction:

$$
\left\{\begin{array}{l}
\hat{x}_{k}=A_{k-1} x_{k-1}+B_{k-1} I_{L, k-1} \\
\hat{P}_{k}=A_{k-1} P_{k-1} A_{k-1}^{T}+Q_{k-1}
\end{array}\right.
$$

Update: 


$$
\left\{\begin{array}{l}
e_{k}=Y_{k}-C_{k} \hat{x}_{k} \\
K_{k}=\hat{P}_{k} C_{k}^{T}\left(C_{k} \hat{P}_{k} C_{k}^{T}+R_{k-1}\right)^{-1} \\
H_{k}=\frac{1}{M} \sum_{i=k-M+1}^{k} e_{i} e_{i}^{T}, R_{k}=H_{k}-C_{k} \hat{P}_{k} C_{k}^{T}, Q_{k}=K_{k} H_{k} K_{k}^{T} . \\
x_{k}=\hat{x}_{k}+K_{k} e_{k} \\
P_{k}=\left(I-K_{k} C_{k}\right) \hat{P}_{k}
\end{array}\right.
$$

$Y_{k}$ is the experimental data. Through the calculation procedure of AEKF, its shortages could be summarized that the AEKF linearized the state equations, which led to systemic high-order truncation errors, and the AEKF had to compute the Jacobian matrix, which caused the calculation complexity.

\section{PAUKF SOC Estimation Algorithm}

The PAUKF was based on the unscented Kalman filter technic which can transfer the mean and covariance values of the battery state that is no need to linearize the state equations of the battery model, so that the higher estimation accuracy can be achieved.

The key technic of UKF is the unscented transformation which needs the roots computation of the covariance matrix that may have the matrix singularity problem because of the rounding errors of the computer. In this paper, this problem could be resolved by the parallel arithmetical technic.

The procedure of PAUKF was shown in (12) (20)

Initialize with:

$$
x_{0}, P_{0}=\left(P_{0, U}, P_{0, S O C}\right), Q_{0}=\left(Q_{0, U}, Q_{0, S O C}\right), R_{0} .(12)
$$

Generating Sigma points:

$$
\begin{aligned}
& \left\{x_{k, i}\right\}=\left\{x_{k}, x_{k}+r \sqrt{P_{k, \bullet}}, x_{k}-r \sqrt{P_{k, .}}\right\},(i=0,1,2) .(13) \\
& \left\{\begin{array}{l}
r=\sqrt{3} \\
w_{i}=\left\{\begin{array}{l}
\frac{2}{3}, i=0 .(14) \\
\frac{1}{6}, i \neq 0
\end{array}\right.
\end{array}\right.
\end{aligned}
$$

\section{Prediction:}

With (5), the prediction of the battery state equations could be achieved as(15).

$$
\left\{\begin{array}{l}
\hat{U}_{p, k}=\sum_{i=0}^{2} w_{i} \times \hat{U}_{p, k, i} \\
S \hat{O} C_{k}=\sum_{i=0}^{2} w_{i} \times S \hat{O O} C_{k, i} \\
\hat{P}_{k, U}=\sum_{i=0}^{2} w_{i}\left(\hat{U}_{p, k, i}-\hat{U}_{p, k}\right) \times\left(\hat{U}_{p, k, i}-\hat{U}_{p, k}\right)^{T}+Q_{k-1, U} \\
\hat{P}_{k, S O C}=\sum_{i=0}^{2} w_{i}\left(S \hat{O} C_{k, i}-S \hat{O} C_{k}\right) \times\left(S \hat{O} C_{k, i}-S \hat{O O C} C_{k}\right)^{T}+Q_{k-1, S O C}
\end{array}\right.
$$

With (6), the prediction of the battery observation equation could be achieved as 


$$
\hat{U}_{T, k}=\sum_{i=0}^{2} w_{i} \hat{U}_{T, k, i}
$$

Update:

$$
\begin{aligned}
& \left\{\begin{array}{l}
P_{U_{T}, U_{T}}=\sum_{i=0}^{2} w_{i}\left(\hat{U}_{T, k, i}-\hat{U}_{T, k}\right)\left(\hat{U}_{T, k, i}-\hat{U}_{T, k}\right)^{T}+R_{k} \\
P_{U_{p}, U_{T}}=\sum_{i=0}^{2} w_{i}\left(\hat{U}_{p, k, i}-\hat{U}_{p, k}\right)\left(\hat{U}_{T, k, i}-\hat{U}_{T, k}\right)^{T} \\
P_{S O C, U_{T}}=\sum_{i=0}^{2} w_{i}\left(S O C_{k, i}-S \hat{O} C_{k}\right)\left(\hat{U}_{T, k, i}-\hat{U}_{T, k}\right)^{T}
\end{array}\right. \\
& \left\{\begin{array}{l}
K_{k, U}=P_{U_{p}, U_{T}} \times P_{U_{T}, U_{T}}^{-1} \\
K_{k, S O C}=P_{S O C, U_{T}} \times P_{U_{T}, U_{T}}^{-1} \\
U_{p, k}=\hat{U}_{p, k}+K_{k, U}\left(U_{T, k}-\hat{U}_{T, k}\right) \\
S O C_{k}=S O \hat{O} C_{k}+K_{k, S O C}\left(U_{T, k}-\hat{U}_{T, k}\right)
\end{array}\right. \\
& \left\{\begin{array}{l}
P_{k, U}=\hat{P}_{k, U}-K_{k, U} P_{U_{T}, U_{T}} K_{k, U}^{T} \\
P_{k, S O C}=\hat{P}_{k, S O C}-K_{k, S O C} P_{U_{T}, U_{T}} K_{k, S O C}^{T} .(19)
\end{array}\right. \\
& \left\{\begin{array}{l}
e_{k}=Y_{k}-\left[-\hat{U}_{p, k}-R_{o} I_{L, k}+U_{o c}\left(S O C_{k}\right)\right] \\
H_{k}=\frac{1}{M} \sum_{i=k-M+1}^{k} e_{i} e_{i}^{T}, R_{k}=H_{k}-C_{k} \hat{P}_{k} C_{k}^{T} .(20) \\
Q_{k, U}=K_{k, U} H_{k} K_{k, U}^{T} \\
Q_{k, S O C}=K_{k, S O C} H_{k} K_{k, S O C}^{T}
\end{array}\right.
\end{aligned}
$$

\section{Experiments and validation}

In this paper, the Federal Urban Driving Schedule (FUDS) was applied to validate the the accuracy and preponderance of PAUKF SOC estimation algorithm.

The FUDS experimental current profile was shown in Fig. 2, which was achieved by the experiment of a electric car.

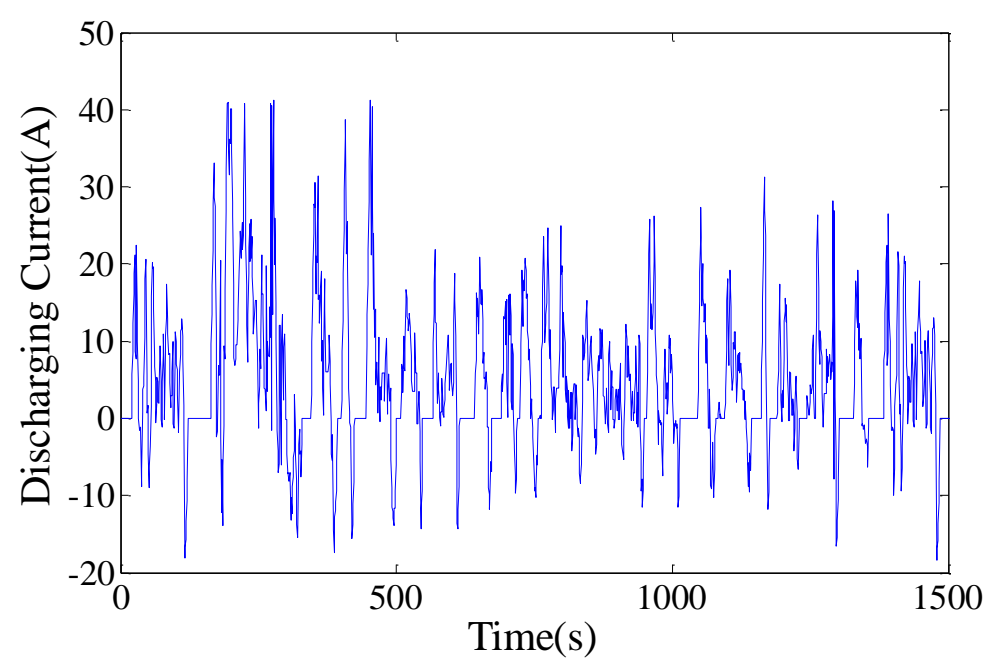

Fig. 2 The FUDS current profile 
The measured terminal voltage, PAUKF terminal voltage estimation and AEKF terminal voltage estimation were shown in Fig. 3. From Fig. 3, the good accuracy of terminal voltage estimation was validated. The real SOC of the battery, PAUKF estimated SOC and AEKF estimated SOC were shown in Fig. 4. The detailed error statistics was given in the Table 1. Obviously, the PAUKF SOC estimation method has higher accuracy and preponderance over AEKF, and the the mean SOC estimationerror is less than $2.4 \%$.

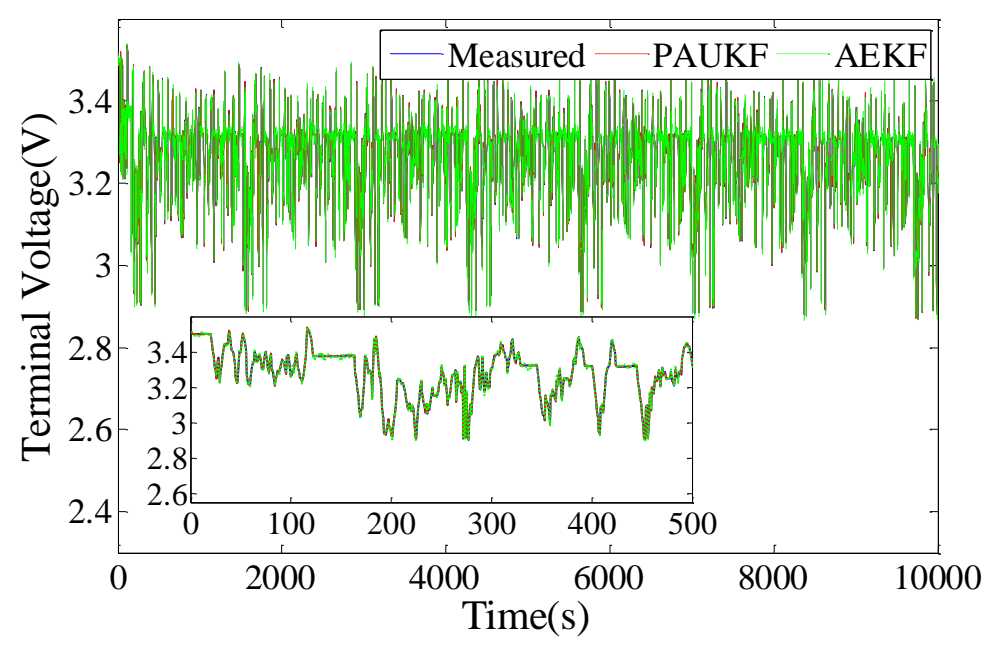

Fig. 3 The Measured and estimated terminal voltage under FUDS

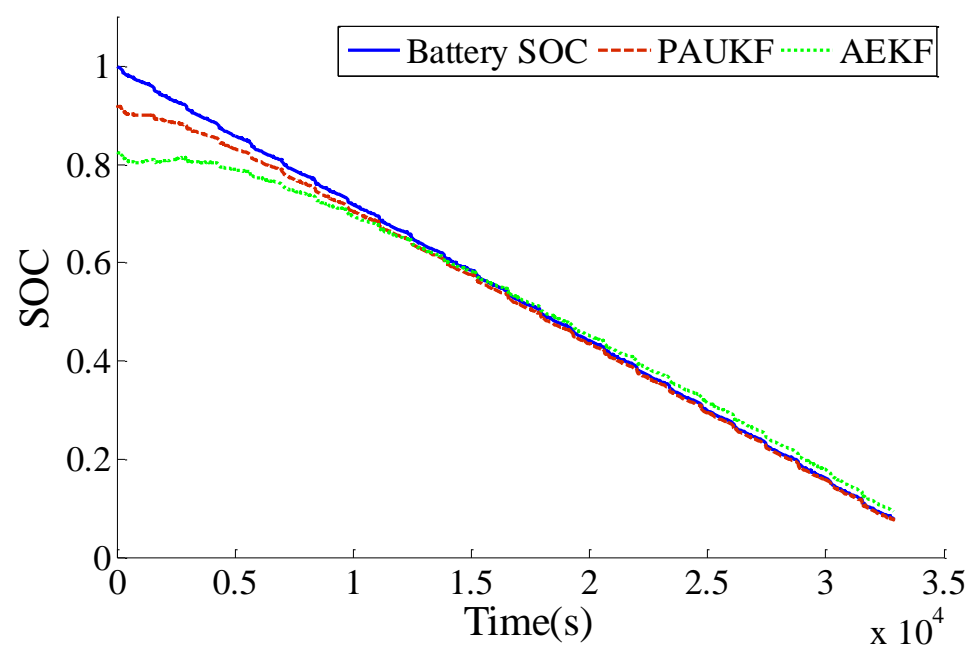

Fig. 4 The estimated SOC with PAUKF and AEKF under FUDS

Table 1 Error statistics analysis

\begin{tabular}{c|c|c}
\hline \multirow{2}{*}{ Algorithm } & \multicolumn{2}{|c}{ Mean Estimation error } \\
\cline { 2 - 3 } & Terminal Voltage $(m V)$ & SOC $(\%)$ \\
\hline PAUKF & 17 & 2.37 \\
AEKF & 25 & 4.74 \\
\hline
\end{tabular}

\section{Conclusions}

In this work, a novel SOC estimation algorithm was presented using the technic of parallel arithmetical unscented Kalman filter. A ELCM battery model and the parameter identification were studied. Then, the AEKF and PAUKF SOC estimation algorithms were proposed and derived, and the disadvantages of AEKF were discussed. Finally, the comparison and validation were presented. The results showed the PAUKF has obviously preponderance over AEKF, of which mean error is less than $2.4 \%$. 


\section{Acknowledgements}

The work of this paper is funded by National Science Foundation Program of China (51307160).

\section{References}

[1] Hong Zhang, Li Zhao, and Yong Chen. Energies. Sci. Vol. 8-12 (2015), p. 13811-13828.

[2] Xuanju Dang, Li Yan, Kai Xu, etc..ElectrochimicaActa. Sci. Vol. 188 (2016), p.356-366.

[3] Xiaopeng Chen, Weixiang Shen, Mingxiang Dai, etc.. IEEE Transactions on Vehicular Technology. Sci. Vol. 65-4 (2016), p. 1936-1947.

[4] JinhaoMeng, Guangzhao Luo, and Fei Gao. IEEE Transactions on Power Electronics. Sci. Vol. 31-3 (2014), p. 2226-2238.

[5] Vigneysh T., Kumarappan N..Internatinational Journal of Hydrogen Energy. Sci. Vol. 41-3 (2015), p. 1877-1891.

[6] Congzhi Liu, Weiqun Liu, Lingyan Wang, etc.. Journal of Power Sources. Sci. Vol. 320 (2016), p. 1-12.

[7] Zhangbao Wei, LimTuti Mariana, Skyllas-Kazacos M., etc.. Applied Energy. Sci. Vol. 172 (2016), p. 169-179.

[8] BartlettAlexander, Marcicki James, Onori, Simona, etc..IEEE Transactions on Control Systems Technology. Sci. Vol. 24-2 (2016), p. 384-399.

[9] XuebingHan,MinggaoOuyang,LanguangLu and JianqiuLi. Journal of Power Sources. Sci. Vol. 278 (2015), p. 814-825.

[10]ZeCheng,JikaoLv, YanliLiu and ZhihaoYan. Journal ofApplied Mathematics. Sci. Vol. 29-1 (2014), p. 221-228. 